\section{Low Uptake of Intermittent Preventive Treatment in Ghana; An Examination of Health System Bottlenecks}

\section{Abstract}

Despite the devastating impact of malaria in pregnancy in Africa, the uptake of intermittent preventive treatment for malaria prophylaxis has remained considerably low. In Ghana, uptake of optimal doses stands (4+ doses) stands at $38.5 \%$ in spite of the fact that antenatal care is around $87.3 \%$. In this paper, a review of existing bottlenecks is assessed to determine the underlying factors affecting optimal uptake in country. The World Health Organisation's health system strengthening framework is adapted to examine these factors. Finally, a health information strategy is proposed to ameliorate the underlying the issues identified.

Received: August 21, 2017, Accepted: August 24, 2017, Published: September 01, 2017

\section{Introduction}

Malaria during pregnancy has unequivocally poor health outcomes to a pregnant woman and the developing foetus [1]. A recent systematic review and meta-analysis of seven trials suggest the association of malaria in pregnancy (MIP) to severe maternal anaemia, cerebral malaria in women and low neonatal birthweight which could result to preterm delivery and eventual death [2]. It is estimated that, globally, 125.2 million pregnant women are at risk of malaria with $81.5 \%$ living in sub-Saharan Africa [3]. Consequently, the sustainable development goals (SDGs) addresses MIP through setting ambitious targets of eradicating malaria among pregnant women, ending neonatal mortality as well as reducing maternal morality ratio to 70 per 100,000 by 2030 [4].

In 2006, as a response to MIP, the World Health Organisation (WHO) recommended tripartite integrated interventions for pregnant women in malaria endemic regions. These were; intermittent preventive treatment using sulfadoxine-pyrimethamine (IPTp-SP), Insecticide mosquito nets (ITNs) and intermittent screening and treatment (IST) [5]. Despite, remarkable successes made towards ITNs and IST, coverage of IPT-SP continues to linger (average of $11.77 \%$ in sub-Saharan Africa) [6].

Intermittent preventive treatment is a dose therapy of an antimalarial drug administered after 16 gestation weeks (quickening) monthly to pregnant women in malaria endemic regions (WHO 2013). In many African countries including Ghana, IPT is administered as a directly observed therapy (DOT) during each scheduled antenatal care (ANC) visit [7].

\section{Emmanuel Nene Odjidja ${ }^{1 *}$, Charles Kwanin² and Manika Saha}

1 Institute of Global Health and Development, Queen Margaret University, Edinburgh UK

2 Nurse, Ghana Adventist Health Services, Ghana

\section{Corresponding author:}

Emmanuel Nene Odjidja

\section{emmaodjidja@gmail.com}

Institute of Global Health and Development, Queen Margaret University, Edinburgh, UK.

Citation: Odjidja EN, Kwanin C, Saha M (2017) Low Uptake of Intermittent Preventive Treatment in Ghana; An Examination of Health System Bottlenecks. Health Syst Policy Res. Vol. 4 No. $3: 58$

Many countries in sub-Saharan Africa continue to experience a huge disparity between ANC visit (75\% average in sub-Saharan Africa) and IPT coverage [6]. This illustrates substantial missed opportunities at ANC clinics [8]. Adopting the WHO's building blocks, this paper will discuss the intricacies of health system challenges relating to the low IPT uptake and further propose a viable solution towards establishing a strong health information framework for capturing and reporting exact impediments that affects the successful implementation of IPT.

\section{Context and Problem statement}

Ghana is situated in West Africa with population and life expectancy of 27.4 million and 61.3 years respectively [9]. In 2014, the World Bank reported that Ghana spent only $2.13 \%$ of its Gross domestic product on health with out-of-pocket spending contributing 26.84\%. Maternal mortality ratio and infant mortality stand at 319 per 100,000 and 41 per 1,000 respectively (missed target for MDG goal 4 and 5) [6]. Estimates further reveal that malaria in pregnancy alone contributes to $9.0 \%$ of maternal deaths and it is the singular highest contributor to all OPD admissions $(197,017$ cases) among pregnant women [10]. The Ghana Health Service adopted SP as the appropriate medicine for IPT in 2003 [11]. In 
2004, it updated the antimalarial drug policy to reflect WHO's guidelines on how IPT should be administered [11]. Although chapter 4 of the antimalarial drug policy clearly stipulates that IPT shall be administered as DOT on monthly basis during ANC visits until delivery [11], available data from the demographic health survey shows a significant discrepancy between ANC coverage (4+ visits) and IPT uptake (3+ doses) (Figure 1). Although Ghana has made remarkable success of closing the deficit between ANC visit and IPT uptake (from $68.8 \%$ in 2003 to $48.8 \%$ in 2014), it is still confronted with over 50,000 pregnant women who are unprotected [6].

\section{Rationale and limitations of using WHO's health System building blocks in the context of malaria research.}

This paper recognises the importance of having an integrated framework that responds to several facets of a health challenge. This reinforces the fact that low IPT uptake is complex and multifaceted which demands a holistic approach in diagnosing the challenges [7]. Employing the WHO systems building blocks would therefore provide a common comprehension of the pertinent attributes engulfing IPT uptake in such a resourceconstrained setting like Ghana.

While the framework provides a coherent understanding of system dynamics related to a health challenge [12], its failure to recognise the critical importance of interaction among the blocks limits the understanding of impacts on a health system [13]. Health systems like any living organism present consistently complex interactions that culminate to new dynamics and trends and as such, demand tailored responses. For example, between 2012 and 2013, WHO updated the IPT policy to increase doses from 3 to 5 till delivery [5], yet tools for capturing data were still 3 doses [14]. Such an inconsistency suggests that it will be worthwhile in learning about the feedback loop and communication patterns among the building blocks within a health system and how the system is continually shaped by these factors.

Missing elements of demand-related issues and behaviour change undermines the weight of each block [13]. This offers an abysmal understanding of input, outputs, outcomes within a health system and how that is interlinked to demand of services of a health system [13]. For example, in learning about IPT uptake, service delivery, and health workforce is much stronger and has a direct outcome on demand-related whereas finance and health information will be classified as inputs. Attaching weights will guide its use in assessing demand-related issues and behavioural change thereafter [13].

\section{Diagnosing Low IPT Uptake; Focus on the Six Building Blocks}

\section{Governance}

According to the Ghana Health Service, private maternity homes contribute to $19 \%$ of total facilities that provide maternal care [15]. The Demographic health survey (2014), also indicates that $8 \%$ of all pregnant women had received $4+$ antenatal care from private facilities yet only $3.34 \%$ of these women received IPT $3+$ which was far lower than the national average of $38.5 \%$. Categorically attributes this difference to weak regulations, compliance to national protocols and adequate oversight by health authorities to enforce existing laws within private facilities.

Again, the section 4.2 of the antimalarial drug policy of Ghana

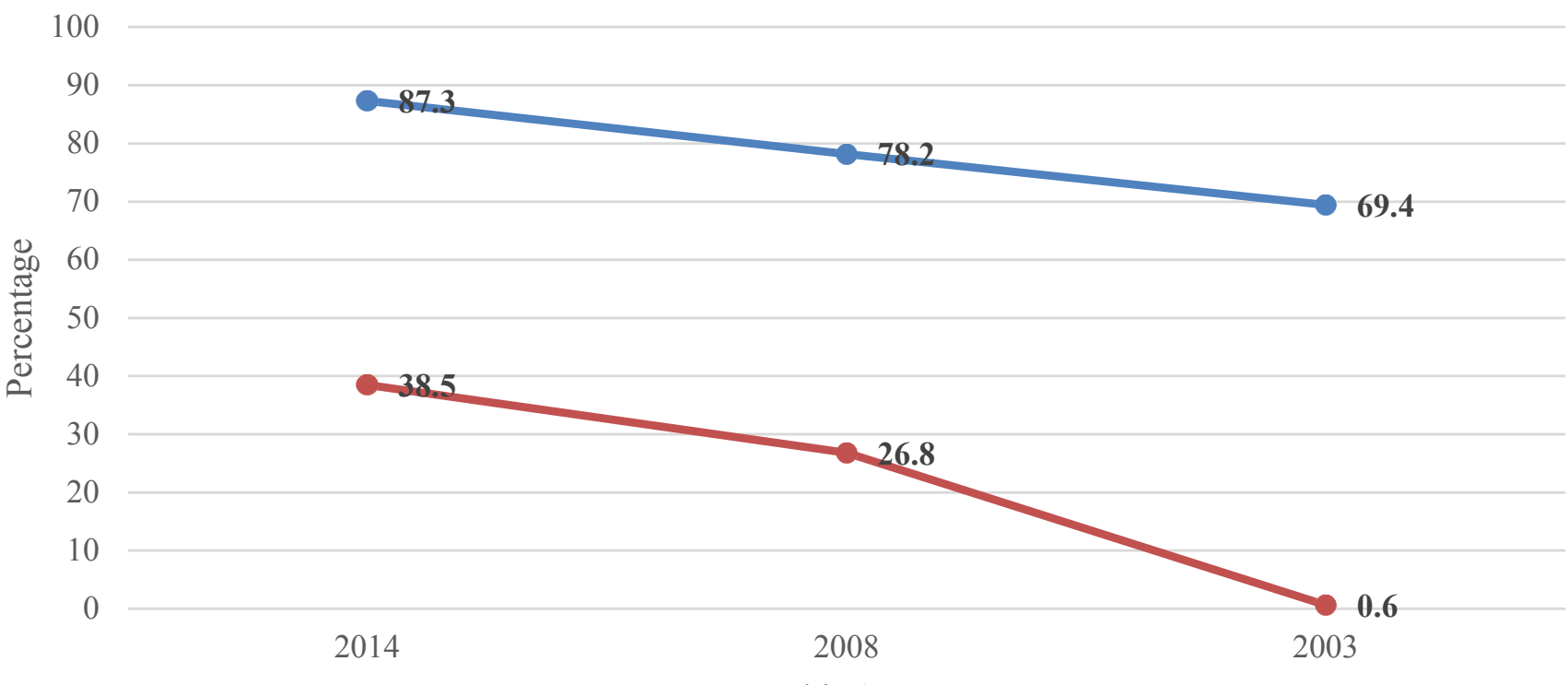

Demographic Survey years

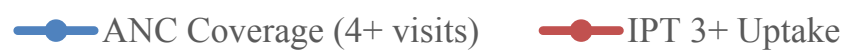

Figure 1 Trend between ANC coverage and IPT 3+ uptake.

Source: DHS survey, 2014 
details the procedures in administering IPT that is limited in adequately adopting the comprehensive recommendations of WHO as updated in 2008. For example, the policy fails to espouse WHO's guidelines on the management of side effects which is among the major reasons for high dropouts [5]. This situation has resulted to health workers discontinuing IPT when side effects manifest after a second dose [16].

\section{Financing}

Although Ghana implemented a free maternal health care policy which removed all forms of official financial barriers in 2003 [17], other financial impediments arising from under-the-table payments and transportation costs continue to hinder utilisation of services [18]. IPT and other ANC interventions are administered in health centres, which are sparsely distributed in the country [18]. Transportation to these inequitably distributed facilities manifests into a barrier, which affects the uptake of IPT among poor women in rural areas. A disaggregation of the demographic health survey by place of residence and wealth (2014) indicates that whereas IPT $3+$ in urban areas was $41.9 \%$, rural areas were trailing with $35.8 \%$. Similarly, pregnant women in the highest wealth quintile had attained coverage of $50.6 \%$ while those at the lowest only reached $36.6 \%$ [6].

Overall, MIP area continues to be among the underinvested areas in developing countries by donors [19]. For example, the US President's Malaria initiative (PMI) Ghana (2015) [20] committed only $\$ 990,000$ (3.54\%) of its operational budget to activities to strengthen IPT uptake in the health system. Undoubtedly, operational MIP research has received chronic underfunding culminating to stagnation in innovation [21]. According to National Malaria Control Program (NMCP) report (2014), although it made significant efforts in executing most of its program activities, two key research pertaining to the impact of IPT and underlying reasons of high IPT dropout had not been carried out due to lack of funds. It is apparent that without a commitment to intensive research to influence behavioural change, an increase in IPT uptake will remain a mirage.

The Government of Ghana's matching funding for the malaria program (NMCP) has been woefully inadequate. Global Fund continues to be the highest donor together with supplements from DFIP, USAID PMI, UNICEF and WHO [10]. This continually threatens the sustainability of MIP efforts, especially after the post-GFATM era.

\section{Health workforce}

Ghana is one of the countries with the severe imbalance in Midwives and nurses (MN) (World Bank, 2010). With an MN ratio of 0.926 midwives to 1000 people [22], Ghana suffers a huge deficit that clearly affects the implementation of programs especially at periphery facilities [10].

There is limited understanding of Ghana's IPT policy among health workers [16]. A study conducted to assess knowledge of health workers according to the national guidelines on dosing, time, restrictions and DOT strategy found that only $47.8 \%$ of health workers have a comprehensive knowledge of the IPT policy [16]. It further reveals that only $49.3 \%$ of midwives and frontline nurses could demonstrate full competence according to the national guidelines. This is very surprising considering the fact that these cadres of health workers play the crucial role in promoting IPT among pregnant women.

\section{Product and technology}

Chico and colleagues have identified supply chain strengthening as a key determinant in preventing periodic stock-outs of SPs. Yet, the supply chain of SPs in Ghana is continually hampered by periodic stock imbalances and periodic stock outs [10]. The National Malaria program although in 2014 purchased and distributed $19,878,570$ tablets to all regions in the country, existing transportation bottlenecks hindered disbursement to periphery facilities in the districts [10]. This culminated to stockouts of SPs within almost every district [10].

Local pharmaceutical companies have limited capacity to meet the growing demand for quality SPs especially among subSaharan countries [7]. According to the NMCP's report, out of all SPs procured, the only local pharmaceutical company supplied could supply only $16.20 \%$ making the program depend on international companies to supply remaining $83 \%$. Considering the bureaucracy involved in importing drugs, the inability of local companies to respond to the demand of SPs results in low responsiveness especially during emergency stock-outs [23]. Also, the growing resistance of SP viz a viz non-existence of a medicinal alternative encourages low uptake especially in instances when resistance is reported [8].

\section{Health Information (Monitoring and Evaluation)}

Tactical use of health information, intelligence and research forms an integral facet of governance and leadership function. The ability of health information systems to capture and report reliable information on health determinants, systems performance and health status improves decision making at every level of authority [12]. This includes standard indicators that provide consistent data. Currently, the main indicator used by donors and government for making IPT interventions is number of women received SP during ANC visits (NMCP report, 2014). This indicator has been misunderstood and captured differently by different studies. For example, the MICS report (2011) pegged it at IPT $2+$ and recorded a $64.6 \%$ whiles the demographic health survey (2014) calculated IPT $3+$ with $38.5 \%$. Such non-standard and inconsistent indicator reporting creates discrepancies that undermine the importance of evidence in decision-making.

Ghana just like most malaria endemic countries lack integrated M\&E systems to capture preventive and therapeutic efficacy of IPT [24]. These are important in detecting and monitoring parasitemia prevalence, safety and pharmacovigilance of antimalarial in pregnant women [24].

Data validation teams play an important role in generating quality data for decision-making. In Ghana, data validation teams are integrated into the district health management teams and they are responsible for ensuring data quality [14]. Despite being vital in ensuring data quality, they are highly inadequate especially 
across rural districts. Reports from USAID facility assessments indicate that only $38 \%$ of districts had functional data validations teams. This obviously questions the quality of data reported on IPT uptake from lower facilities.

\section{Service delivery}

Health systems are strengthened to deliver safe and quality services with a goal of improving health outcomes [12]. Service delivery of IPT in Ghana is compounded with accessibility and practicality challenges, socio-cultural barriers as well as general relationships that exist between providers and users [25] According to the Demographic health survey, there are $13.8 \%$ of pregnant women who still do not have access to ANC. Late presentation to ANC clinics also plays a significant factor to low IPT uptake [26]. A study found that the median for first ANC visits was 3.6 months with rural dwellers even dipping further to 3.7 months [6]. Given the fact that IPT is administered after the first trimester of pregnancy, late presentation thereof will affect the achievement of $3+$ uptakes.

Existing relationship between providers and clients together with perceived low quality of ANC services influences users' choice and target of ANC [25]. A study in Ashanti region of Ghana found that pregnant women's preference to seek care from traditional birth attendants was due to perceived unfriendly and rude behaviour of professional health workers as against welcoming TBAs [27].

Again, Mubyazi and his colleagues indicate that although SPs were available in facilities, the absence of essential utilities such as disposal cups and clean drinking water constrained its implementation as DOT.

\section{Proposed strategy towards strengthening Health Information (M\&E) Systems for IPT uptake}

A robust health information system is one that detects, communicates and contains events that threaten public health including emergencies [12]. To this end, timeliness and precision are key. Ghana must invest immensely into an electronic data capture at every level of data entry that will aggregate real-time data into the existing district health management information systems (DHMIMs). This will allow health managers to make swift and timely interventions especially during emergencies and SP stock outs.

The research community, WHO Ghana and the ministry of Health should collaborate to establish M\&E plans for the growing SP resistance and its impact on IPT effectiveness. Furthermore, the malaria technical working group must readjust sentinel sites to MIP parasitemia transmission especially in hard-to-reach rural areas as well as exact data on the predictors (social, economic and cultural) that influence ANC uptake [7].

Independent data validation teams must be instituted and empowered at each administrative level (from facility to district to regional and national). Again, to motivate validation teams, Ghana should consider adopting Rwanda's performance based financing [28]. With the growing preference for electronic data systems, validations teams can validate real-time data either remotely or virtually [29].
Finally, stakeholders' and donors must reinforce their commitment to monitoring and evaluation through working in coordination. To make judicious use of resources, donors must agree to consolidate efforts and produce data that is consistent, reliable and timely. For example, instead of conducting DHS by USAID and multiple indicator cluster surveys (MICS) by UNICEF, there should be a consolidated survey that would take place in shorter intervals to understand the landscape better [30].

\section{Conclusion}

Although Ghana has made quite significant strides in improving IPT uptake from $26.8 \%$ in 2008 to $38.5 \%$ in 2014 (second highest after Zambia) (DHS 2014), there is still the need for Government to Ghana to recommit itself towards rigorously pursuing the fight against MIP. With an ambitious SDG target of reducing maternal mortality rate (MMR) from 319 to 70 per 100,000 live births, Ghana must dedicate itself to increasing overall health budget from $8.90 \%$ to meet the Abuja declaration minimum of $15 \%$. Again, in ensuring sustainability of the malaria program, giant steps should be taken towards attaining a 50-50 disease split funding between government and development assistance. Finally, policy and practice must be consistent at all times to ensure service delivery follows latest and updated guidelines. This also signifies that Ghana should make essential investments in enhancing the feedback loop between managers and frontline workers. More importantly, training institutions should seek and adopt latest MIP guidelines during instruction.

\section{Competing Interest}

We declare no competing interests.

\section{References}

1 Desai M, Ter Kuile FO, Nosten F, Mcgready R, Asamoa K, et al. (2007) Epidemiology and burden of malaria in pregnancy. Lancet Infect Dis 7: 93-104.

2 Kayentao K, Garner P, Van Eijk AM, Naidoo I, Roper C, et al. (2013) Intermittent preventive therapy for malaria during pregnancy using 2 vs 3 or more doses of Sulfadoxine-Pyrimethamine and risk of low birth weight in Africa. Systematic review and Meta-analysis. JAMA 309: 594-604.

3 Dellicour S, Tatem AJ, Guerra CA, Snow RW, Ter Kuile FO, et al. (2010) Quantifying the number of pregnancies at risk of malaria in 2007: A demographic study. PLoS Medicine 7: e1000221.

4 Friedrich MJ (2016) Sustainable development goals launched. JAMA 315: 647.

5 World Health Organisation (2013) WHO policy brief for the implementation of intermittent preventive treatment of malaria in pregnancy using sulfadoxine-pyrimethamine (IPTp-SP). Geneva, Switzerland.

6 Demographic Health Survey (2014) Demographic Health Survey dataset. USAID.

7 Chico RM, Dellicour S, Roman E, Mangiaterra V, Coleman J, et al. (2015) Global call to action: Maximize the public health impact of intermittent preventive treatment of malaria in pregnancy in subSaharan Africa. Malaria Journal Vol 14: 207. 
8 Hill J, Kazembe P (2006) Reaching the Abuja target for intermittent preventive treatment of malaria in pregnancy in African women: A review of progress and operational challenges. Trop Med Int Health 11: 409-418.

9 The World Bank Group (2016) Maternal mortality ratio (modeled estimate, per 100, 000 live births). Geneva.

10 National Malaria Control Program (2014) Annual report national malaria control programme. Ghana: Ghana Health Service.

11 Ghana Health Service (2010) Anti-Malaria drug policy for Ghana. Accra, Ghana.

12 WHO (2007) Everybody's business: Strengthening health systems to improve health outcomes: WHO's framework for action. [1 Edn] Geneva: World Health Organization.

13 Mounier-Jack S, Griffiths UK, Closser S, Burchett H, Marchal B (2014) Measuring the health systems impact of disease control programmes: A critical reflection on the WHO building blocks framework. BMC Public Health 14: 278.

14 Amoakoh-Coleman M, Kayode GA, Brown-DaviesC, Agyepong IA, Grobbee DE, et al. (2015) Completeness and accuracy of data transfer of routine maternal health services data in the greater Accra region. BMC Research Notes Vol 8: 114.

15 Anemena S, Martey M, Nortey L, Kontor K, Adams I, et al. (2012) Private health sector development policy (Rhsd). Accra, Ghana: Ghana Health Service.

16 Smith Paintain L, Antwi GD, Jones C, Amoako E, Adjei RO, et al. (2011) Intermittent screening and treatment versus intermittent preventive treatment of malaria in pregnancy: Provider knowledge and acceptability. PLoS ONE 6; e24035.

17 Witter S, Arhinful DK, Kusi A, Zakariah-Akoto S (2007) The experience of Ghana in implementing a user fee exemption policy to provide free delivery care. Reprod Health Matters 15: 61-71.

18 Ganle J, Parker M, Fitzpatrick R, Otupiri E (2014) A Qualitative study of health system barriers to accessibility and utilization of maternal and new-born healthcare services in Ghana after user-fee abolition. BMC Pregnancy and Childbirth 14: 425.

19 Pigott DM, Atun R, Moyes CL, Hay SI, Gething PW, et al. (2012)
Funding for malaria control 2006-2010: A comprehensive global assessment. Malar J 11: 246.

20 USAID and USAID Global Health, CDC, Dept. Of State and Dept. Of Health and Human Resources (2014) President's Malaria Initiative Ghana malaria operational plan FY 2015. Accra, Ghana.

21 Greenwood B, Alonso P, Ter Kuile FO, Hill J, Steketee RW, et al. (2007) Malaria in pregnancy: Priorities for research. Lancet Infect Dis 7: 169-174.

22 The World Bank Group (2010) Nurses and midwives (per 1, 000 people). World Health Organization's Global Health Workforce Statistics, OECD, supplemented by country data.

23 Rassi C, Graham K, Mufubenga P, King R, Meier J, Gudoi SS (2016) Assessing supply-side barriers to uptake of intermittent preventive treatment for malaria in pregnancy: A qualitative study and document and record review in two regions of Uganda. Malaria Journal 15: 1.

24 Brabin BJ, Wasame M, Uddenfeldt-Wort U, Dellicour S, Hill J, et al. (2008) Monitoring and evaluation of malaria in pregnancy developing a rational basis for control. Malaria Journal 7: S6.

25 Mubyazi GM, Magnussen P, Goodman C, Bygbjerg IC, Olsen OE, et al (2008) Implementing intermittent preventive treatment for malaria in pregnancy: Review of prospects, achievements, challenges and agenda for Research 2007-11-27. Open Trop Med J 1: 92-100.

26 Pell C, Menaca A, Were F, Afrah NA, Chatio S, et al. (2013) Factors affecting Antenatal care attendance: Results from qualitative studies in Ghana, Kenya and Malawi. PLoS ONE 8: e53747.

27 Dufie AG (2010) Factors influencing the uptake of intermittent preventive treatment of malaria in pregnancy in the Bosomtwe district of Ghana.

28 Rusa L, Fritsche G (2007) Second edition Rwanda: Performancebased financing in health. In: Health Financing.

29 Mutale W, Chintu N, Amoroso C, Awoonor-Williams K, Phillips J, et al. (2013) Improving health information systems for decision making across five sub-Saharan African countries: Implementation strategies from the African health initiative. BMC Health Serv Res 13: S9.

30 Ministry of Finance (2016) Summary of expenditure by SubProgramme, Economic Item and Funding - Health. Accra: Ministry of finance, Ghana. 